\title{
Man: The Profound Change
}

\author{
Almada $\mathrm{F}^{1}$, Fernando $\mathrm{C}^{2}$, Lopes $\mathrm{H}^{2}$, Vicente $\mathrm{A}^{* 3}$ \\ ${ }^{1}$ Retired University Professor / Independent Researcher, Portugal \\ ${ }^{2}$ University of Madeira, Portugal \\ ${ }^{3}$ University of Beira Interior, Portugal \\ *Corresponding author: António Vicente, University of Beira Interior, Rua Marquês D’Ávila e Bolama, Portugal \\ To Cite This Article: António Vicente. Man: The Profound Change. Am J Biomed Sci \& Res. 2019 - 6(1). AJBSR.MS.ID.000997. DOI: 10.34297/ \\ AJBSR.2019.06.000997.
}

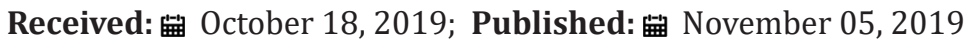

\section{Mini Review}

A statement: A profound change knocks at the door!

People's reaction - condescending smiles and shout: We've all seen it, we all know it!

But it is not true. A profound change is in fact at our door, if it has not already begun to enter the house and the spirit, but few have seen it and still less have perceived it.

In any case, resistances to change are expected, which in a succinct way, we will say whether they are consciously assumed or not, can be due to:

a) the natural fear of innovation and everything that is new.

b) to the installed interests and the fear of losing stewardship

c) the incommensurability between paradigms that Kuhn had already talked about.

\section{Sport: A Tool and an Instrument}

Sport is a structuring phenomenon in any society nowadays. With great weight in the lives of individuals and societies, just see the space that it occupies in the media and in the conversations and debates between people, sport has gained a dimension that is reflected in all its aspects. From economics to education, from health to industry, from spectacle to tourism, sport is a cornerstone in generating dynamics and policies, in creating movements and in stopping processes, in overcoming difficulties and as a means of celebration. Sport, at the same time, takes on positions that seemingly and superficially might seem contradictory. It can thus be a factor of stability and simultaneously have a function of transformation mean for the same individuals or social groups. But it will only be able to perform all these functions efficiently if it is understood beyond its immediate and light aspects, if it is understood in its functionalities and the active principles (the consequences that it triggers) that it may have, the side effects that it can have, the fidelity and safety on the information about it, the knowledge of the dosages to be used, the value and the difficulties of the prescriptions that are activated, the objectives and cautions to be taken in the management of all this process.

If something that is anodyne can be used without great concern, since it is innocuous (although the innocuous is always a function of the weight and measure of what is used, that it is only innocuous to a reasonable size of its use) the effects we can expect from its use are zero or very low. Sport, as we have just presented, is far from innocuous. The results it triggers are varied, profound and with high effects on short, medium and long term.

The great strength of sport as an action instrument is also a risk factor of the many and varied risks that it can have. Risks that are further exacerbated by interests that always appear associated with sport, such as the financial values involved, the visibility it produces, the emotions it arouses, the economic weight it has, the motivations it awakens, the transformations it causes in individuals and in societies, the sensitivities that blunted, an infinity of forms of intervention that sport can have and that make it precious, but also dangerous.

It is not easy to deal with this problem with Intellectual honesty, but it is fundamental to define what we know, what we do not know, what we should know and what we have the obligation to know.

Observers may not be neutral (they never are, because they interpret the facts, and overcome Popper who asserted that " $a$ fact is a fact", considering Kuhn who said that "...a fact is a fact, but its interpreting depends on the paradigm in which it is made...") but that they should strive to be exempt and be able to surf the emotions without letting themselves be rolled up, has therefore an arduous but fundamental work, so that the fruits can be harvested 
and used by who knows how to give them good use. Thus, in order to respect the identity of man, personalization of the pedagogical process is necessary, which requires that sports producers, at all levels of decision-making and in the different fields of intervention, must at least have undergraduate training, which allows them to be able to act in a diagnostic, prescription and control logic and not through the application of pre-established standards and the reproduction of stereotypes, the result of their experiences or the "recipes" of some enlightened ones.

However, producers of sport with higher education (doctorate, master's and bachelors degree) are not being able to impose themselves in the different professional fields, and are often losing space for intervention, which is not acceptable if they effectively dominate one of the more powerful tools of power that Toffler talked: the knowledge.

\section{Sport, games and rupture problems with which they are debating nowadays}

\section{About some characteristics of sport:}

a. Sport as a factor of stability - where sport serves as a buffer against confrontations and conflicts (sometimes creating even less aggressive or less serious alternative conflicts).

b. Sport as a factor of transformation - where sport leads to new paths and prepares people (sportsmen, but also spectators, scholars, etc.) to be able to look for alternatives and / or enjoy the innovation.

c. Sport is a mean, a tool, and not an end on itself. But the result, the quest for victory, is important because it is the guarantee that the opposition stands firm and will seek a performance as strong as possible and that an assessment of the performances of the actors in the process is constantly carried out.

d. Accepting defeat does not mean giving up the victory, but it is a warning that haven't been reached yet the level needed to achieve a victory. Winning, in turn, means that higher levels can be tried to test the levels achieved

e. Understanding sport is fundamental to assess whether the individual's own training will not have to be called into question, since the removal of the lines of operation that allow us to take advantage of such a rich environment as sport can originate in the ignorance of what is sought, the inability to achieve it or even to be dragged by emotions that constitute a motivation for the exercise of the activity and a grid of control and evaluation of the level reached by the sportsman.

\section{About some situations on sport}

a. What is a false start in a sprint race in athletics? When the meaningful difference begins to be around the tenth of a second, as in a start of a sprint race in athletics, it is essential to characterize (more accurately) the phenomenon in order to manage it. A tenth of a second is a huge time for the existing capacity to measure, but in relation to the functioning of the human muscle a 'infinitesimal time'. It is no longer enough to measure the strength made in the starting blocks, the decision should be made at the beginning of the work of the muscle groups involved.

b. What will a car race mean in a world where no one will drive the car that carries it? Will it be a mere random drawing (dice roll or roulette) or will other forms of performance be created by the driver (who is going to be replaced by a robotic system)?

\section{About some dynamic and their reflex on sport}

1. The limits on drug control and the possibilities that open up for its use, but which lead to a rethinking of the whole process in order to be able to take advantage of the activity (the current ones, or possibly others that can be defined and preferred for the advantages they can bring).

2. Bionic man and fairness in sports competitions - contrary to what many claim sports is not a competition between equals and equity will hardly cease to be a mere expression of good intentions. All individuals are different and competing demands not fairness but the ability to evaluate oneself and others or circumstances so as to try to establish appropriate strategies (not always for victory but sometimes for a less intense defeat), for the exercise of the operational frameworks to be used, for the diagnosis of the evolutions that must be attempted and, of course, for the evaluation of all these conjectures and ways of acting.

3. Sport can be a mere instrument of 'lucky draw' - a substitute for 'rolling a dice' or rolling a roulette wheel. But in these cases the advantages hardly over the costs.

4. Like drugs, the adaptations achieved at the genetic level may contribute to specific performances. But what advantages can we draw from such a process? The simple pleasure of being able to cheat or, for example, the possibility of conducting other types of experiences? Which? At what costs? With what advantages?

\section{Some Paradoxes that Confront Us Today}

1. Today is unanimously accepted the need to customize training, each individual has its own characteristics that must be enhanced in order to achieve the most profitable balance for him. However, although this statement of intentions leads many to already make a diagnosis (which in itself could even be considered an evolution), this diagnosis continues to be made on the basis of a set of "critical components" of the gesture (ie the means - in a static framework and without understanding the established dialectics), trying to find what the individual 
lacks in order to "fit" into the pattern, instead of, depending on a functional view of the activity, looking for opportunities to improve that individual.

2. The previous point is self-explanatory, because if the knowledge of sports could not move to a dynamic picture (which sometimes happens even with those who have a training that would require it, if updated), a better answer than the 'empirical instincts' is possible which sometimes even succeed but fail to achieve continuities that successively reinforce incomes.

3. Knowledge, today, allows us to perceive an organic man in which the functionality is made of dialectics much closer to the real than some models that we may already consider traditional. The paradox is that the extraordinary financial value involved (maturities, premiums, 'parallel' financial circuits, etc.) which often blocks what could be done in less favored situations could be possible.

\section{Some Profound Changes, Breakdowns in the Social Structure in the Past}

This is a difficult subject not only because of the variety of options and interpretations, because of the vastness of the theme, but even more because the overlapping of the observer with the phenomenon demands an intellectual honesty in areas where emotion normally dominates.

1. The first world globalization resulting from the period of the Portuguese Discoveries represents a rupture and a profound change. A profound change not because it has gone from a Mediterranean world (from a European perspective) to a space encompassing almost (not the Arctic) the world's oceans.

The fundamental happened because the conditions reached (suffered?) led to readjustments and transformations that forced new ways of living, of feeling and of thinking that led to the transmutations that still today we are trying to adapt ourselves:

1. We have a similar situation to the previous one in a more schematic and succinct way in another break that we are living at the level of food. Again from a perspective of what is sometimes called the 'western world' we have passed, after World War II, so a little over seventy years ago, of a world of scarcity and lack of food to meet the food needs of the general population (we do not consider the punctual cases that naturally exist because of organizational or other similar deficiencies) to a world of abundance.

We went, schematically, from a world in which there was a potato for five people to another in which there are five potatoes for one person. What happened? Having failed to make the necessary adjustments to the diet and the accompanying organizations we have, again schematically, two potatoes per person to eat, two to make vodka and one to spoil. Obesity and alcoholism are mere indicators of a structural failure resulting from a mismatch that has not been corrected yet. Again, it was not the material aspects and the new means available, but the way of placing man in the process that leads to costs that would have been expendable if there had been a concern to think a little. In the impossibility of showing to who cannot see, of making to listen what they cannot hear, of making to feel what they cannot feel... but considering the important theme we have left some notes for reflection on three aspects, examples with which we seek to illustrate our position:

1. Sport, games and rupture problems with which they are debating nowadays

2. Some paradoxes that confront us today

3. Some profound changes, breakdowns in the social structure in the past [1-4].

The analysis and validation of both the knowledge that underlies all the processes in which sport is involved and even for the use of a framework explaining its functionality, even if it is in a very simplified and schematic way as follows:

\section{Stimulus / Aggression " " Reaction» " Adaptation " 》 Transformation}

That show the evolutionary process of any living being in contact with its context (in all or in some aspects - physiological, psychological, sociological, etc.).

\section{Conclusion}

When we think of change, the profound changes that we are experiencing in our daily lives, we tend to think of the enormous amount of 'toys' that have been presented to us since the beginning of the industrial revolution. An amount (and quality) that is still increasing overwhelmingly. Like any other spoiled boys, not knowing what to do with all this gifts, tired with plenty, staying on the superficiality of the uses and eager for emotions that may be considered strong (the strength of the emotions depends, of course, not only on the intensity of the stimulus, but also the sensibility of those who suffer the effect of this stimulus), we seek to complement the simplicity found in satisfying our needs and desires with the search for ghosts and catastrophes with which we always lived .even when the pressure of everyday life led us to ignore them pressured as were with more immediate needs. Sport, like any other 'toy', tool or instrument, is available for the different processes and jobs that it may serve. Deep change knocks at the door. It's up to us what's going to happen next. Many things will change, but it will always be man (rather than the 'little' toys we like to play with) the determinant of the change that is going to (or is) happen(ing).

\section{References}

1. Almada F, Fernando C, Lopes H, Vicente A (2018) Operationalization of the 'Human Body Domain': A Structural and Functional Conception. J Phy Fit Treatment \& Sports 4(2). 
2. Almada F, Fernando C, Lopes H, Vicente A (2018) The Understanding and Explanation of Sport: The Importance of Methodology. Biomedical Journal of Scientific \& Technical Research 10(4).

3. Almada F, Fernando C, Lopes H, Vicente A (2019) Health, Sport, Education and... the rest. Biomedical Journal of Scientific \& Technical Research 16(3).
4. Almada F, Fernando C, Lopes H, Vicente A (2019) For a Vision of Man in a Dynamic Framework: Promote Equilibium Versus Add or Remove 'Parts' - Facts, Strategies and Operational Modes. Orthopaedic Surgery and Traumatology 2(5): 407-416. 